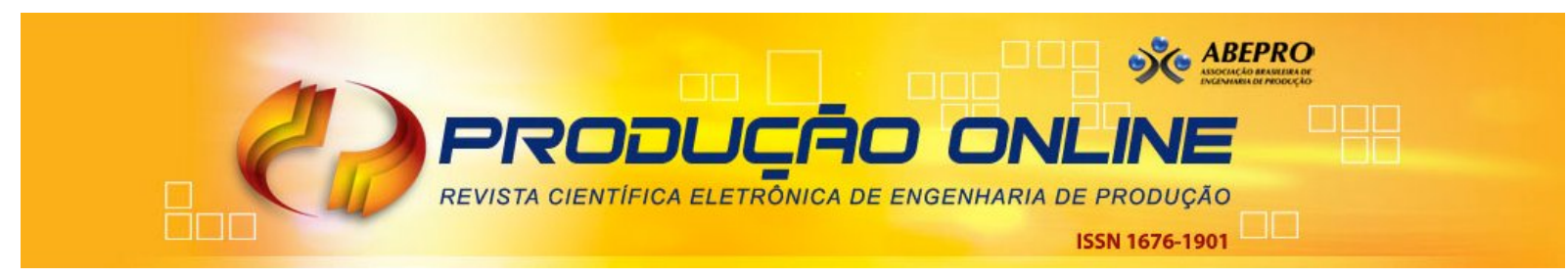

\title{
TEORIA DE OPÇÕES REAIS: AVALIAÇÃO ECONÔMICO-FINANCEIRA DE PROJETOS NA INDÚSTRIA CERÂMICA
}

\section{REAL OPTIONS THEORY: FINANCIAL-ECONOMIC ASSESSMENT OF PROJECTS IN THE CERAMICS INDUSTRY}

\author{
Léony Luis Lopes Negrão* E-mail: leony@uepa.br \\ Mariana Pereira Carneiro* E-mail: mariana karneiro@yahoo.com.br \\ Jorge de Araújo Ichihara* E-mail: Jorge.ichihara@hotmail.com \\ Rubens Cardoso da Silva* E-mail: rubens@cardoso.eng.br \\ *Universidade do Estado do Pará (UEPA), Belém, PA
}

\begin{abstract}
Resumo: $O$ presente estudo teve como objetivo avaliar economicamente a implantação do Mecanismo de Desenvolvimento Limpo na substituição de biomassa não renovável por biomassa renovável no processo produtivo de uma empresa de cerâmica vermelha. A avaliação pretendeu demonstrar o valor da flexibilidade gerencial de Diferimento conforme adaptações realizadas na metodologia proposta por Copeland \& Antikarov (2001), a qual adiciona à avaliação tradicional de fluxo de caixa descontado a Opção Real - OR. Essa determinação seguiu um roteiro de etapas essenciais para a análise das variáveis que compreendem o modelo e que possibilitou a ordenação dos resultados quanto aos valores da OR considerada e do valor presente incluindo a flexibilidade gerencial. Finalmente, como resultado deste estudo concluiu-se que a Teoria de Opções Reais por meio da Opção de Diferimento ou Adiamento contribuiu com informações que auxiliaram nas decisões gerenciais de investimento em projetos quando comparados à metodologia tradicional de avaliação visto que são consideradas incertezas inerentes ao projeto, tal como o ambiente real.
\end{abstract}

Palavras-Chave: Opções Reais. Indústria Cerâmica. Crédito de Carbono. Viabilidade Econômica. Opção de Diferimento

Abstract: The present study aimed to evaluate economically the implementation of Clean Development Mechanism in the substitution of non-renewable biomass to renewable biomass in the production process of a red ceramics enterprise. The evaluation intended to show the value of management flexibility, according to adaptations made in the methodology proposed by Copeland \& Antikarov (2001), adding to the traditional discounted cash flow the evaluation the Real Options. This procedure follows a routine of essential steps for the analysis of variables that comprises the model and enabled the ordination of the results based both on the Real Options and the present values, including management flexibility evaluation. It could be concluded that Real Options Theory, and the Option to Delay or Postponement contributed with information that might assist and improve projects investment decisions, since several real-world inherent uncertainties are considered.

Keywords: Real Options. Ceramics Industry. Carbon Credit. Economical Evalution. Option to Delay

Revista Produção Online, Florianópolis, SC, v.15, n. 3, p. 1049-1079, jul./set. 2015. 


\section{INTRODUÇÃO}

Os créditos de carbono surgiram nos países desenvolvidos como meio de inibir a produção de poluentes. A legislação daqueles países estabeleceu níveis máximos de emissões de certos gases e aqueles que conseguissem manter o volume de emissões abaixo do estabelecido tinham direito a títulos de créditos equivalentes à porção reduzida, os quais podiam ser vendidos àqueles que não tiveram o mesmo sucesso, e que não deveriam infringir as regras. Assim os que realizaram investimentos para melhorar a qualidade de seus processos operacionais teriam uma recompensa e, por outro lado, aqueles que não o fizeram ou não o fizeram em montante suficiente teriam que pagar pela poluição produzida. Com o advento do Protocolo de Quioto, esse mecanismo foi aperfeiçoado e adotado (RIBEIRO, 2005).

Os créditos de carbono são gerados principalmente por meio da implementação dos chamados Mecanismos de Desenvolvimento Limpo - MDL. Entende-se MDL como: medidas que possibilitam a redução de emissões de gases que causam o efeito estufa durante o processo de produção, gerando "Certificado de Redução de Emissão - RCEs".

Para efeitos do MDL, compreende-se por atividades de projeto (project activities) as atividades integrantes de um empreendimento que tenham por objeto a redução de emissões de gases de efeito estufa e/ou a remoção de $\mathrm{CO}_{2}$ (LOPES, 2002).

Para que um projeto resulte em RCEs, as atividades de projeto do MDL devem, necessariamente, passar pelas etapas do ciclo do projeto, que são oito: estudo de viabilidade; elaboração de documento de concepção de projeto (DCP), que usa metodologia de linha de base e plano de monitoramento aprovados; validação (esta verifica se o projeto está em conformidade com a regulamentação do Protocolo de Quioto); aprovação pela Autoridade Nacional Designada - AND -, que no caso do Brasil é a Comissão Interministerial de Mudança Global do Clima CIMGC - (analisa a contribuição do projeto para o desenvolvimento sustentável); submissão ao Conselho Executivo para registro; monitoramento; 
verificação/certificação; e emissão de unidades segundo o acordo de projeto (MERLIN, 2007).

Entretanto, implantar projetos de MDL requer investimentos que necessitam de indicadores que sinalizam ou subsidia às decisões quantos aos aspectos financeiros e econômicos do mesmo.

A abordagem clássica de análise de viabilidade econômica de projetos, ao assumir fluxos de caixa pré-determinados, pode ser caracterizada como estática, pois ignora a possibilidade da adoção de decisões gerenciais. Conforme Copeland e Antikarov (2001), "o Valor Presente Líquido - VPL - pressupõe implicitamente que não há flexibilidade na tomada de decisões." Essa afirmação está fundamentada no fato de que o método do Fluxo de Caixa Descontado - FCD - é aplicado em um determinado momento do tempo, quando todos os fluxos de caixa relacionados ao projeto são definidos e mantidos fixos ao longo de toda a vida útil do projeto. Assim, não são incorporados os valores das novas decisões tomadas a posteriori a sua posterior mensuração no instante "zero".

Para contemplar essa flexibilidade gerencial existem os métodos de precificação de opções. Essa aplicação gera a Teoria de Opções Reais - TOR. A Opção Real é o direito, mas não a obrigação, de empreender uma ação a um custo pré-determinado por um período de pré-estabelecido (COPELAND; ANTIKAROV, 2001).

Dias (2004) observa que a abordagem das OR é uma ferramenta para avaliação de projetos sob incerteza. Dixit e Pindyck (1998) afirmam que, analogamente às Opções Financeiras, para as OR a oportunidade de investir é uma opção de compra, assim como a oportunidade de desinvestimento é uma opção de venda. Ainda segundo Dixit e Pindyck (1998), o exercício ótimo da OR ocorre quando o VPL obtido é grande o suficiente frente aos custos por esperar por mais informações. Brandão (2002) acrescenta que para determinado projeto possuir valor para OR, deve embutir três características: o investimento necessário deverá ser total ou parcialmente irreversível, ter flexibilidade que admita adiamento, suspensão, ampliação e abandono e, também, que os Fluxos de Caixas futuros sejam incertos.

Em adição a essa conclusão gerencial, na visão da área de Estratégia Empresarial, existem fatores estratégicos fundamentais para a manutenção ou 
aumento da competitividade de uma empresa, sem a qual sua lucratividade futura (e, em última análise, a sua própria sobrevivência) ficará comprometida.

Tais fatores podem não ser convenientemente traduzidos em incrementos dos fluxos de caixa futuros. Assim sendo, projetos para os quais se calcula Valor Presente Líquido - VPL - baixo, ou mesmo negativo, podem ser aceitos por razões estratégicas, e projetos com VPL aparentemente altos podem ser ignorados, por não atenderem aos objetivos estratégicos da empresa.

A TOR é promissora para a análise de viabilidade econômica e estratégica de projetos, mas sua operacionalização é complexa; proliferam estudos de simplificação em Matemática, Estatística e Informática. Ainda são poucos os softwares robustos e de interface fácil com o usuário. Daí a importância de artigos que mostram aplicações bem-sucedidas que possam constituir caminhos de raciocínio lógico para o desenvolvimento de sistemas informatizados.

O presente artigo possui o objetivo realizar a avaliação econômica da implantação do Mecanismo de Desenvolvimento Limpo em uma empresa de cerâmica vermelha, à luz de metodologia da Teoria de Opções Reais, em que foi considerada a Opção de Diferimento ou Adiamento.

Entre as fases do processo produtivo das empresas de cerâmica vermelha se evidenciam o uso de lenhas, oriundas da floresta nativa ou dos resíduos das indústrias madeireiras, como combustível a ser empregado nos fornos das cerâmicas para cozimento dos produtos fabricados. A geração e disponibilização desordenada desse insumo sempre sofreu questionamento quanto à legalidade de sua origem e disponibilidade para gerações futuras - insumos não renováveis -, e quanto à identificação de outros materiais - insumos renováveis - que possam substituir aquele.

\section{MODELOS DE AVALIAÇÃO COM RISCO}

Neste tópico, busca-se abordar o custo de capital como modelo de avaliação que se enquadram no conceito genérico do fluxo de caixa descontado, acrescentando à tradicional análise do VPL o conceito de risco. 


\subsection{Abordagem da Taxa de Descontado Ajustada}

Assumiu-se até então um universo livre de riscos e incertezas no cálculo do VPL em alternativas de investimento. Esta suposição é claramente muito distante da realidade das decisões de investimentos, e ressalta a necessidade de se incorporar o conceito de risco na análise de investimentos através dos métodos do fluxo de caixa descontado.

Ao introduzir incertezas, a mesma idéia básica de decisão do VPL permanece inalterada, porém o conceito de "investimentos compatíveis" passa a significar investimentos com as mesmas características de risco. Conforme sugerido por Trigeorgis (1996), a taxa de desconto "r" passa ser substituída por "k", que representa a soma de $r$ (taxa de juros livre de risco) mais um prêmio de risco "p" usado para compensar o risco associado ao projeto. A equação 01 descreve o VPL.

$V P L=-I+\sum_{t=1}^{T} \frac{E\left(F C_{t}\right)}{(1+k)^{t}}$

$I$ = investimento requerido no projeto.

$F C t=$ fluxo de caixa no período " $t$ ".

$\mathrm{T}=$ prazo de vida do ativo.

$k=$ taxa de desconto ajustada ao risco.

A decisão de investir é tomada a partir da seguinte regra (WOILER; MATHIAS, 1997).

Se VPL > 0; Investe.

Se VPL < 0; Não Investe.

Se VPL = 0; Indiferente entre investir ou não.

\subsubsection{Custo médio ponderado de capital (WACC)}

O Custo Médio Ponderado do Capital (Weighted Average Cost of Capital) WACC - é a média ponderada dos custos marginais do capital após o desconto dos impostos que incidem sobre o mesmo. É adequado ao desconto do fluxo de caixa da empresa ou projeto para fazer pagamentos às fontes de capital próprio e de 
terceiros (COPELAND; ANTIKAROV, 2001). E que pode ser obtido conforme expressão 02.

$$
\begin{aligned}
& \text { WACC }=k_{s} \frac{S}{B+S}+k_{b}(1-I R) \frac{B}{B+S} \\
& \mathrm{~K}_{\mathrm{s}}=\text { custo do capital próprio; } \\
& \mathrm{S}=\text { valor presente do capital próprio; } \\
& \mathrm{K}_{\mathrm{b}}=\text { custo do capital de terceiros; } \\
& \mathrm{B}=\text { valor presente do capital de terceiros; } \\
& \mathrm{IR}=\text { taxa de alíquota do IR. }
\end{aligned}
$$

Sendo que o valor presente do capital próprio (S) é obtido, segundo Copeland e Antikarov (2001), descontando o fluxo de caixa livre ao custo do capital próprio $\left(K_{s}\right)$, vide equação 03. E o valor presente do capital de terceiros (B) será o total de despesa anual em juros descontado pelo custo do capital de terceiros $\left(\mathrm{K}_{\mathrm{b}}\right)$, expressão 04.

$$
\begin{aligned}
S & =\frac{F C F}{k_{s}} \\
F C F & =\text { fluxo de caixa livre; } \\
\mathrm{K}_{\mathrm{s}} & =\text { custo do capital próprio; }
\end{aligned}
$$

$$
B=\frac{J}{k b}
$$

$\mathrm{J}=$ total anual de despesas com juros; $\mathrm{K}_{\mathrm{b}}=$ custo do capital de terceiros;

O cálculo do custo do capital de terceiros $\left(\mathrm{k}_{\mathrm{b}}\right)$ é o resultado das taxas de mercado e da precificação do mesmo para os títulos emitidos pela empresa. Entre os fatores que influenciam o custo do capital de terceiros estão o nível corrente da taxa de juros, o risco de inadimplência da empresa e os benefícios fiscais associados aos empréstimos. Além disso, uma vez que os custos com capital são dedutíveis para fins de imposto de renda, o cálculo do mesmo deve ser ajustado para refletir este efeito (COPELAND; ANTIKAROV, 2001; TRIGEORGIS, 1996).

Para o cálculo do custo do capital próprio $\left(\mathrm{K}_{\mathrm{s}}\right)$ é preciso proceder algumas aproximações que podem ser feitas através do cálculo do valor presente dos dividendos futuros esperados, ou de metodologias que buscam estimar o custo do Revista Produção Online, Florianópolis, SC, v.15, n. 3, p. 1049-1079, jul./set. 2015. 
capital próprio considerando o risco do mercado, como o Modelo de Formação de Preço de Ativos de Capital (Capital Asset Pricing Model) - CAPM - (COPELAND; ANTIKAROV, 2001; TRIGEORGIS, 1996).

\subsubsection{Modelo de formação de preço de ativos de capital (CAPM)}

O Modelo de Formação de Preço de Ativos de Capital - CAPM - oferece uma metodologia atrelada ao conceito de diversificação do risco para relacionar o retorno esperado de um ativo individual ao risco da carteira que representa o mercado, baseado na correlação existente entre o retorno de um ativo e o retorno do mercado como um todo (COPELAND; ANTIKAROV, 2001; TRIGEORGIS, 1996).

Ao revisar o modelo CAPM, Trigeorgis (1996) lista as seguintes premissas fundamentais para a utilização do beta como o único fator para a determinação da compensação pelo risco (prêmio):

$\checkmark$ Investidores são reacionais, e o seu objetivo é a maximização da utilidade esperada de sua riqueza ao final de um único período;

$\checkmark$ Investidores são avessos ao risco e diversificam suas carteiras eficientemente com base na média e na variância do retorno da carteira;

$\checkmark$ Investidores possuem expectativas homogêneas, ou seja, estimativas idênticas dos valores esperados, variâncias, covariâncias, e retornos por ativos de riscos;

$\checkmark$ Existe uma taxa de juros livre de risco $r_{t}$ a qual os investidores podem emprestar ou fazer empréstimos a qualquer momento;

$\checkmark$ Não existem impostos ou custos de transação, e os custos de falência são imateriais; além disso, toda informação está livremente disponível aos investidores;

$\checkmark$ Todos os ativos são perfeitamente divisíveis e líquidos;

$\checkmark$ O mercado é competitivo, de tal forma que os investidores sabem que não são capazes de influenciar o preço ou o montante de ativos com base em suas ações. 
$\mathrm{Na}$ prática, apesar da dificuldade de obtenção de betas com alto grau de precisão, o CAPM oferece uma medida aceitável e amplamente utilizada para a medida de risco para um ativo (ROSS; WESTERFIELD; JAFFE, 1995; SHARPE, 1970). Seu cálculo segue a expressão 05.

$$
\begin{aligned}
& K_{e}=r_{f}+\beta\left(E\left(r_{m}\right)-r_{f}\right) \\
& r_{f}=\text { taxa livre de risco; } \\
& \beta=\text { risco sistêmico do capital próprio; } \\
& E\left(r_{m}\right)=\text { é o retorno esperado da carteira de mercado. }
\end{aligned}
$$

\subsection{Abordagem da Taxa de Desconto Neutra de Risco}

A estimativa do valor de um projeto pode ser obtida tanto ao se descontar os fluxos futuros de caixa pela taxa de desconto ajustada ao risco quanto pelo desconto dos fluxos de caixa previamente ajustada ao risco pela taxa de juros livre de risco. Ambos os métodos devem levar ao mesmo resultado. Dessa forma calcula-se o VPL pelo método da taxa de juros ajustada ao risco, conforme a expressão 06 (COPELAND; ANTIKAROV, 2001).

$$
V P L=-I+V P \quad \therefore \quad V P=\frac{E(F C F)}{1+r_{f}+\left(E\left(r_{m}\right)-r_{f}\right) \beta}
$$

O método da taxa neutra de risco apresenta os mesmos resultados, porém pela sua metodologia, o ajuste é feito no numerador, ao invés do denominador. De acordo com o CAPM, o beta entre uma empresa, ou projeto, e o mercado é definido pela expressão 07 (COPELAND; ANTIKAROV, 2001).

$$
\beta=\frac{\operatorname{COV}\left(k_{e}, r_{m}\right)}{\operatorname{VAR}\left(r_{m}\right)}
$$


Se substituir ke por seu equivalente na equação do valor presente de um único período, têm-se VP conforme expressão 08 (COPELAND; ANTIKAROV, 2001).

$$
V P=\frac{E(F C F)}{\left(1+k_{e}\right)} \quad \therefore \quad k_{e}=\frac{E(F C F)}{V P}-1
$$

Substituindo na definição de beta para incluir o retorno de um período, obtémse a nova definição, expressão 09 (COPELAND; ANTIKAROV, 2001).

$$
\beta=\frac{\operatorname{COV}\left[\frac{E(F C F)}{V P}-1, r_{m}\right]}{\operatorname{VAR}\left(r_{m}\right)}=\frac{1}{V P}\left[\frac{\operatorname{COV}\left(E(F C F), r_{m}\right.}{\operatorname{VAR}\left(r_{m}\right)}\right]
$$

Substituindo o valor de beta na equação do valor presente em um período pela expressão 09, obtém-se a nova equação (10) do valor presente (COPELAND; ANTIKAROV, 2001).

$$
V P=\frac{E(F C F)}{1+r_{f}+\left(E\left(r_{m}\right)-r_{f}\right)\left(\frac{1}{V P}\right)\left[\frac{\operatorname{COV}\left(E(F C F), r_{m}\right)}{V A R\left(r_{m}\right)}\right]}
$$

Por fim, pelo CAPM, o preço de mercado do risco é igual a $\frac{\left\lfloor E\left(r_{m}\right)-r_{f}\right\rfloor}{\operatorname{VAR}\left(r_{m}\right)}$, e simbolizando-o por $\lambda$, reescreve-se a equação do valor presente conforme expressão 11.

$$
V P=\frac{E(F C F)-\lambda C O V\left[E(F C F), r_{m}\right]}{1+r_{f}}
$$

Copeland e Antikarov (2001) afirmam que esta abordagem faz o ajustamento do risco, subtraindo uma perda dos fluxos monetários esperados, a fim de obter, 
primeiramente, os fluxos de caixa em termos de certeza equivalente, para em seguida, descontá-los à taxa livre de risco.

\section{MODELOS DE PRECIFICAÇÃO DE OPÇÕES}

Qualquer um dos principais modelos de avaliação do valor do preço de uma opção (binomial e Black-Scholes) necessariamente utilizam um conjunto básico de variáveis que necessitam ser conhecidas ou estimadas para que possam fornecer o preço da opção. Segundo Copeland e Antikarov (2001) as variáveis são tais quais:

$\checkmark$ Preço do ativo subjacente (S): é o preço de mercado do ativo sobre o qual a opção de compra ou venda é baseada em um dado momento. Em opções reais é o valor do projeto (VP);

$\checkmark$ Preço de exercício (X): é o preço pelo qual o detentor da opção pode exercê-la. Em opções reais é o valor do investimento do projeto no instante zero;

$\checkmark$ Tempo até o vencimento (T): fração anual do prazo de vencimento da opção;

$\checkmark$ Taxas de juros (r): é a taxa de juros que influi na determinação do preço da opção;

$\checkmark$ Volatilidade $(\sigma)$ : é o movimento que sofre o ativo subjacente com o passar do tempo. Indica a incerteza (ou risco) quanto aos retornos proporcionais por este ativo.

Dentre os itens apresentados, as quatro primeiras variáveis são intuitivas e auto-explicativas. No entanto, a volatilidade $(\sigma)$ é a mais difícil de ser determinada, pois não é diretamente observada e precisa, portanto, ser estimada.

A volatilidade histórica é medida normalmente pelo desvio padrão dos movimentos no preço do ativo subjacente no passado, expressa em percentual, e calculada, na maioria das vezes, para períodos pequenos e recentes. Na prática, todavia, há investidores que utilizam períodos mais longos o até mesmo a análise gráfica.

Silva Neto (1996) apresenta os efeitos das variabilidades nas variáveis sobre o preço de uma opção. O que segue na tabela 3.1.

Revista Produção Online, Florianópolis, SC, v.15, n. 3, p. 1049-1079, jul./set. 2015. 
Tabela 3.1 - Efeitos das mudanças nas variáveis sobre o preço de uma opção

\begin{tabular}{ccc}
\hline Movimento da variável & Efeito no valor da call & Efeito no valor da put \\
\hline Ativo objeto aumenta & Aumenta & Diminui \\
Ativo objeto diminui & Diminui & Aumenta \\
Volatilidade aumenta & Aumenta & Aumenta \\
Volatilidade diminui & Diminui & Diminui \\
Passagem do tempo & Diminui & Diminui \\
Taxa de juros aumenta & Diminui & Diminui \\
Taxa de juros diminui & Aumenta & Aumenta \\
\hline
\end{tabular}

Fonte - Adaptado de Monteiro (2003)

\subsection{Modelo Binomial}

O modelo binomial é visualmente mais simples e intuitivo para a avaliação do preço de opção. Devido a essa vantagem gráfica o modelo binomial tem sido também o modelo mais utilizado por praticantes que buscam nas opções uma forma de gerenciamento de seus investimentos em ativos reais.

O modelo binomial desenvolvido por Cox, Ross e Rubinstein (1979), assume que o preço de determinado ativo $S$ pode assumir no momento dt um valor $S u$, com probabilidade de ocorrência $p$ e um valor $S d$ com probabilidade de ocorrência 1- $p$. No momento 2dt o ativo $S$ poderá assumir três valores: $S u^{2}$, Sud ou $S d^{2}$. Conforme pode ser verificado na figura 3.1.

Na figura, $u$ e $d$ correspondem aos fatores de subida e descida de preços, respectivamente. $S$ é o preço do ativo no instante $t=0$.

Os fatores de subida e descida, respectivamente, são dados pelas equações 11 e 12.

$$
\mathrm{u}=\mathrm{e}^{\sigma \sqrt{\mathrm{d} t}} \quad(12) \quad \mathrm{d}=\mathrm{u}^{-1}
$$

$\mathrm{dt}=\mathrm{T} / \mathrm{n}$, onde $n$ é o número de passos na árvore entre os instantes inicial e final. 
$p=\frac{e^{r d t}-d}{u-d} \quad$ se o ativo não pagar dividendos.

Figura 3.1 - Modelo Binomial

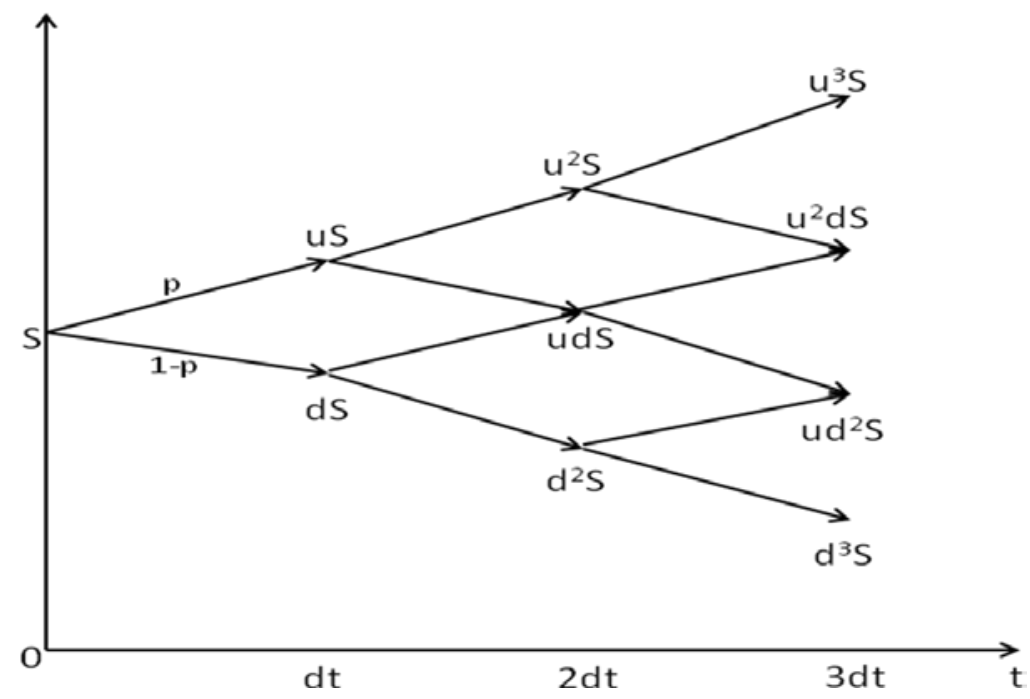

Fonte: Adaptado de Cox, Ross e Rubinstein (1979)

Essa mesma proposição é utilizada por Copeland e Antikarov (2001) na avaliação da evolução dos preços em tempo discreto, segundo a multiplicação do mesmo por $u$, no movimento ascendente, e $d$, no descente, ainda considerada a probabilidade $p$ para o movimento ascendente e 1-p para o movimento descendente.

O estudo revela ainda que os movimentos ascendentes e descendentes seriam proporcionais à volatilidade do projeto.

\section{OPÇÕES REAIS (REAL OPTIONS)}

A avaliação por Opções Reais - OR - deve ser considerada como extensão da avaliação por VPL sem flexibilidade. Segundo a proposição de Black e Scholes (1973) apud Dixit e Pindyck (1998), fundamenta-se a teoria desenvolvida para avaliação de Opções Financeiras. Essa teoria permite, porém, uma única fonte de incerteza e uma única Opção.

A abordagem da teoria das Opções Financeiras é então expandida para a avaliação que compreenda diversas incertezas, que permita múltiplas Opções e que esteja associado ao ativo real, a OR. A terminologia OR foi utilizada por se tratar de 
avaliação de Opções relacionadas a ativos reais, enquanto que Opções, isoladamente, representam ativos financeiros (COPELAND; ANTIKAROV, 2001).

Os autores Dias (2004); Dixit e Pindyck (1998); Brandão, Dyer e Hahn (2005) acrescentam que para determinado projeto possuir valor em OR, deve embutir três características: o investimento necessário deverá ser total ou parcialmente irreversível; ter flexibilidade que admita adiamento, suspensão, ampliação e abandono; e, também, que os fluxos de caixa futuros sejam incertos.

Assim como nas Opções Financeiras, essa flexibilidade gerencial aumenta o valor da oportunidade de investimento em um projeto expandindo seu potencial de ganho bem como limita as perdas relativamente às expectativas iniciais do gerenciamento passivo. Essa expansão no valor da oportunidade de investimento é refletida pelo VPL expandido (VPLE), dado pela soma do VPL estático (VPLS), do projeto sem essa flexibilidade, com o valor da opção de flexibilidade operacional, vide expressão 15 (TRIGEORGIS, 1996).

VPLE = VPLS + valor da opção

Para o cálculo do valor da Opção, pode-se utilizar o processo de Copeland e Antikarov (2001), que possui quatro passos, quais sejam:

a) Análise padrão do valor presente com o emprego de técnicas tradicionais;

b) Construção de uma árvore de eventos, alicerçada em um conjunto de incertezas combinadas que influenciam a volatilidade do projeto;

c) Determinação das decisões gerenciais a serem tomadas nos nós das árvores de eventos, para transformá-los em uma árvore de decisão; e

d) Avaliação dos retornos da árvore de decisão obtidos, aplicando-se os portfólios replicados ou as probabilidades neutras em relação ao risco.

Existem diversos tipos de Opções Reais. Trigeorgis (1996); Copeland e Antikariv (2001) listam alguns que ocorrem naturalmente nos investimentos (opções de adiar, contrair, suspender temporariamente e abandonar o investimento) e outros que podem ser planejados e empreendidos a um custo adicional (opções de expandir e opções compostas, por exemplo). 


\subsection{Opção de Diferimento ou de Adiar o Investimento}

A Opção de adiar ou diferir o investimento dá à administração o direito, mas não a obrigação, de realizar o investimento. Assim, um projeto que pode ser adiado tem mais valor do que o mesmo sem a flexibilidade do adiamento. Isto porque esta Opção permite ao administrador beneficiar-se de movimentos favoráveis ao valor do projeto (como aumento de preços do produto a ser gerado), e evitar perdas caso os cenários desfavoráveis ocorram.

Se a decisão for investir agora, o investidor exerce a Opção e incorrerá um custo de oportunidade igual ao valor da Opção. Por outro lado, a Opção pode ser preservada até o ano seguinte, exercendo-a caso a taxa de atratividade do projeto aumente. Evidentemente, as firmas nem sempre têm essa possibilidade, principalmente em mercados com alto poder concorrencial. A firma deve sempre comparar o custo de adiar - o risco de entrada de novas firmas no mercado ou a perda de fluxos de caixa - com os benefícios de esperar informação nova para subsidiar a decisão de investir (COPELAND; ANTIKAROV, 2001).

Sendo VP o valor presente dos fluxos de caixa futuros, os investimentos realizados no instante t somado ao prêmio da Opção no mesmo instante, possibilita a regra de decisão conforme as expressões 16 e 17 (COPELAND; ANTIKAROV, 2001).

Se VP > I + Ct; investir (Exercer a Opção)

Se VP < I + Ct; não investir (Não Exercer a Opção)

\section{O MÉTODO DE SOLUÇÃO PROPOSTO}

Nas seções anteriores foi apresentada a base teórica da pesquisa, incluindo conteúdos básicos para o entendimento da Teoria de Opções Reais. Contudo, para atingir os objetivos do trabalho, é necessário seguir um encadeamento de idéias e uma sistemática de utilização de informações que caracterizam o método de solução do problema de pesquisa, o qual é abordado nesse capítulo. 


\subsection{Premissas do Método}

Importante ressaltar que as condições de contorno consideradas para este método são:

$\checkmark$ A utilização do modelo de Custo Médio Ponderado de Capital (WACC) para calcular a taxa de desconto;

$\checkmark$ O custo do capital próprio é definido conforme a abordagem do Modelo de Formação de Ativos de Capital (CAPM);

$\checkmark$ A utilização de uma única volatilidade para calcular os valores de VP no decorrer do período do projeto;

$\checkmark$ O modelo binomial é considerado para a estruturação da árvore de eventos a qual o VP do projeto evolui no decorrer do tempo;

\subsection{Etapas do Método de Solução Proposto}

O método apresentado neste capítulo constitui um caso particular do método de Copeland e Antikarov (2001), ao considerar o conceito de volatilidade consolidada àquela que associa duas ou mais volatilidades inerentes ao projeto, e ao analisar uma única volatilidade por todos os períodos de tempo (vide $4^{\mathrm{a}}$ etapa do método), e está divido em seis etapas.

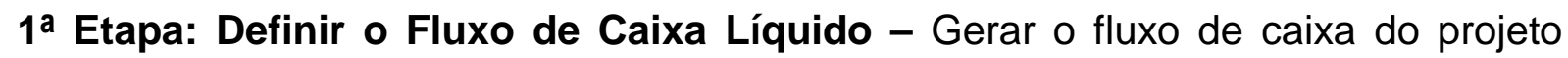
considerando os totais de investimentos, no instante zero, necessários para implantação do projeto; e as receitas futuras deduzidas dos gastos futuros totais no decorrer do período com o funcionamento da empresa; o que gerará o fluxo de caixa líquido futuro.

2a Etapa: Calcular a Taxa de Desconto - Calcular o taxa de desconto que deverá ser utilizada no cálculo do valor presente sem flexibilidade ( $3^{\mathrm{a}}$ etapa). O cálculo é realizado por meio do método do Custo Médio Ponderado de Capital (WACC) conforme a expressão 18. 


$$
W A C C=K_{e} \times\left(\frac{V P_{c p}}{V P_{c p}+V P_{c t}}\right)+K_{b} \times(1+I R) \times\left(\frac{V P_{c t}}{V P_{c t}+V P_{c p}}\right)
$$

$K_{e}$ : Custo do capital próprio;

$V P_{c p}$ : Valor presente do capital próprio;

$V P_{c t}:$ Valor presente do capital de terceiro;

$K_{b}$ : Custo do capital de terceiro;

IR: Alíquota do imposto de renda.

Para projetos que possuem somente capital próprio, concluiu-se que o WACC é igual ao custo do capital próprio que será obtido conforme o Modelo de Formação de Ativos de Capital - CAPM - apresentado na expressão 05.

A proposta metodológica para o cálculo do custo de capital no Brasil apresentada por Assaf Neto (2004) considera a necessidade de apurar o custo de oportunidade dos investidores brasileiros utilizando o benchmark de uma economia mais estável.

Acrescenta-se ao modelo do CAPM, o risco país, que procura retratar o risco da economia de um país. Ao se obter o custo de oportunidade do capital próprio, tendo-se como referência o mercado dos Estados Unidos, deve-se acrescentar ao $K_{e}$ calculado essa taxa de risco da economia. Assim a equação do CAPM para o cálculo do custo do capital próprio no Brasil é expressa pela equação 19.

$$
K_{e}=r_{f}+\beta\left(r_{m}-r_{f}\right)+\alpha_{B R}
$$

rf: Taxa livre de risco;

$\beta$ : Risco sistêmico da ação que corresponde ao risco do capital próprio;

$\left(r_{m}-r_{f}\right)$ : Prêmio pelo risco mercado;

$\sigma_{\mathrm{BR}}$ : Risco país.

$3^{\circ}$ Etapa: Calcular o VP sem Flexibilidade - Obter o VP sem flexibilidade do projeto, descontando as receitas futuras líquidas em cada período por meio do Fluxo de Caixa Descontado, considerando o Custo Médio Ponderado de Capital - WACC - como a taxa de desconto do referido fluxo. 
$4^{\circ}$ Etapa: Calcular a Volatilidade Consolidada do Projeto - Nessa etapa, buscar a identificação das incertezas mais significativas para a obtenção da volatilidade consolidada sob a qual o projeto é submetido no decorrer do tempo.

$5^{\circ}$ Etapa: Modelar a Árvore Binomial - Elaborar a árvore de eventos, com a qual é possível prever os movimentos ascendentes e descendentes do valor do projeto em cada nó da árvore. Segundo as variáveis $u$, (ascendente), e $d$, (descendente), a taxa de desconto livre de risco $\left(r_{f}\right)$, as probabilidades neutras em relação ao risco $p$ e (1$p)$, volatilidade do projeto $(\sigma)$, e finalmente considerando intervalos de tempo de 01 (um) ano (COX, ROSS E RUBINSTEIN 1979). As expressões para essas variáveis são as 20 e 21.

$$
\begin{aligned}
& V P_{n} \text { ascendente }=V P_{n-1} \times u \\
& V P_{n} \text { descendente }=V P_{n-1} \times d
\end{aligned}
$$

É utilizada a árvore binomial, conforme condição de contorno supracitada, segundo Brandão, Dyer e Hahn (2005) para a análise que envolva muitos períodos, a árvore binomial é visualmente simples e permite maior flexibilidade para inserção de OR. Além disso, conforme Hull (2005), a taxa livre de risco é utilizada.

$6^{\circ}$ Etapa: Calcular as Opções Reais Consideradas no Projeto - À árvore de eventos obtida, acrescentar as OR previstas no projeto e projetar uma nova árvore como os valores das Opções consideradas em cada projeto. A nova árvore de decisão representa um valor de flexibilidade gerencial, ou OR, que poderá ser exercida ou não, conforme o tipo de Opção de cada projeto.

\section{AVALIAÇÃO DA IMPLANTAÇÃO DO MDL EM UMA CERÂMICA INDUSTRIAL}

Para realizar a avaliação econômica de implantação do MDL, analisou-se os parâmetros de uma empresa do Arranjo Produtivo Local de Cerâmica Vermelha de São Miguel do Guamá/PA. Considerou-se inicialmente as entradas e saídas provenientes da implantação do projeto, sem flexibilidade. Isso permitiu obter o valor 
do VPL sem flexibilidade e, posteriormente foi inserida a OR de Diferimento ou Adiamento.

O projeto a ser analisado consistiu da substituição da lenha nativa, uma biomassa não renovável, por uma biomassa renovável, sendo que todos os procedimentos necessários para tal se deram formalmente por meio do MDL. Essa substituição possibilitou o registro das reduções de emissões de dióxido de carbono - CO2 - para serem comercializados no mercado de carbono conforme o Protocolo de Quioto, o qual considera a Redução Certificada de Emissões - RCE -, e/ou no mercado voluntário por meio de Verificação de Emissões Reduzidas - VER.

O estudo baseou-se na metodologia proposta por Copeland e Antikarov (2001) a qual foi adaptada para atendimento das especificidades do objeto de estudo. Os passos previstos consideram análise em tempo discreto, permitindo a identificação do comportamento do projeto a cada período de tempo, o que no caso desta pesquisa corresponde a um ano.

\subsection{Gastos e Receitas Operacionais}

Os investimentos necessários à implantação do MDL foram divididos entre investimentos fixos, no valor de $€ 182.283,52$, e consultoria para implantação do mecanismo no valor de €62.207,66. O que segue apresentado no fluxo de caixa do projeto, apêndice 1, e obtido por meio de entrevista com o empresário e análise de documentos na empresa.

As reduções estimadas de dióxido de carbono - CO2 - emitidas na atmosfera conforme definido ao final da implantação do MDL são apresentadas na tabela 6.1. Sendo que, apenas os valores de reduções de 2009 a 2013 foram considerados neste trabalho. 
Tabela 6.1 - Estimativa de redução de emissões durante o período de crédito

\begin{tabular}{|c|c|}
\hline Year & $\begin{array}{l}\text { Annual estimate of emission } \\
\text { reductions per ton of CO2equ }\end{array}$ \\
\hline 2008 & 31,644 \\
\hline 2009 & 40,686 \\
\hline 2010 & 40,686 \\
\hline 2011 & 40,686 \\
\hline 2012 & 40,686 \\
\hline 2013 & 40,686 \\
\hline 2014 & 40,686 \\
\hline 2015 & 40,686 \\
\hline 2016 & 40,686 \\
\hline 2017 & 40,686 \\
\hline Total of estimate reductions (tons of $\mathrm{CO}_{2} \mathrm{equ}$ ) & 397,819 \\
\hline Number of years of the crediting period & 10 \\
\hline $\begin{array}{l}\text { Annual average of estimate reductions for the } \\
10 \text { year crediting period (tons of } \mathrm{CO}_{2} \mathrm{equ} \text { ) }\end{array}$ & 39,782 \\
\hline
\end{tabular}

Fonte: Clean Development Mechanism - Project Design Document Form (2008)

Para o cálculo das receitas futuras que constam no fluxo de caixa do referido projeto, foi considerada a quantidade de redução de $\mathrm{CO}_{2}$ e e o preço da tonelada de dióxido de carbono equivalente $-\mathrm{TCO}_{2} \mathrm{e}-$, obtido por meio da Simulação de Monte Carlo atendendo aos parâmetros estatísticos de distribuição de probabilidade resultante da análise desses preços históricos.

\subsection{Cálculo da taxa de desconto}

Como a empresa utilizou somente capital próprio $\left(K_{e}\right)$ para a implantação do MDL, definiu-se o Custo Médio Ponderado de Capital (WACC) que foi obtido por meio do Modelo de Formação de Preço de Ativos de Capital (CAPM), conforme a expressão 26 (ASSAF NETO, 2004).

A empresa objeto desta pesquisa se caracterizou como sendo de pequeno porte e de capital fechado. O que dificultou a utilização de parâmetros relacionados Revista Produção Online, Florianópolis, SC, v.15, n. 3, p. 1049-1079, jul./set. 2015. 
ao risco, tanto de mercado como sistêmico, onde estes são definidos segundo indicadores de empresas de capital aberto e de grande porte.

Contudo, a taxa livre de risco $\left(r_{f}\right)$ seguiu o método de benchmark. Já o prêmio de risco total $\left(\beta\left(r_{m}-r_{f}\right)+\alpha_{B R}\right)$ que foi somado à taxa livre de risco levou em consideração a proposta desenvolvida por Martelanc (1998) na determinação da taxa de desconto para companhias que não operam na bolsa de valores. Ambos os parâmetros estão descritos tais como:

$\checkmark r_{f}$ é a taxa livre de risco que não pode revelar incerteza alguma e que para esta pesquisa foi considera a média da caderneta de poupança do período de 2009 a 2013, e que resultou no valor de 6,65\% ao ano, cotação em 04/12/2014 conforme 0 site http://www.portalbrasil.net/poupanca mensal.htm, acessado em 04/12/2014; $\checkmark$ O risco sistêmico teve como parâmetro as escalas de valores definidas por Schilt (1982) apud Martelanc (1998) conforme tabela 6.2, e que para esta pesquisa foi adotada a categoria 4 com prêmio total de risco no valor de 21\%; visto que a empresa em questão é de pequeno porte, dependente da habilidade gerencial do proprietário, e que os riscos que envolvem o negócio podem ser considerados, entre outros, os incentivos políticos, fatores ambientais e sociais e a adaptação da estrutura dos custos operacionais às exigências do mercado. 
Tabela 6.2 - Prêmio de risco proposto por Schilt

\begin{tabular}{|c|c|c|}
\hline Categorias & Descrição da Empresa & Prêmio de Risco \\
\hline 1 & $\begin{array}{l}\text { Empresa já estabelecida no mercado com forte posição, boa } \\
\text { administração, nível ótimo de financiamento, passado estável de } \\
\text { lucros, perspectiva otimista de resultados futuros. }\end{array}$ & $6 \%-10 \%$ \\
\hline 2 & $\begin{array}{l}\text { Empresa já estabelecida em ambiente mais competitivo, bem } \\
\text { financiada, boa administração, passado estável de lucros, mas os } \\
\text { resultados futuros são incertos. }\end{array}$ & $11 \%-15 \%$ \\
\hline 3 & $\begin{array}{l}\text { Empresa instalada em mercado altamente competitivo, pouco capital } \\
\text { investido, administração fraca, apesar de boa história de lucros } \\
\text { passados. }\end{array}$ & $16 \%-20 \%$ \\
\hline 4 & $\begin{array}{l}\text { Pequena empresa que depende das habilidades gerenciais de uma ou } \\
\text { duas pessoas, ou empresa de grande porte de natureza cíclica em seus } \\
\text { negócios. Nos dois casos, os resultados positivos futuros são incertos. }\end{array}$ & $21 \%-25 \%$ \\
\hline 5 & $\begin{array}{l}\text { Pequena empresa, dependente de um único proprietário, com lucros } \\
\text { baste incertos. }\end{array}$ & $26 \%-30 \%$ \\
\hline
\end{tabular}

Fonte: Martelanc (1998)

Realizados todos esses procedimentos, chegou-se ao valor do custo de capital com a soma da taxa livre de risco ao prêmio de risco total:

$$
W A C C=27,65 \%
$$

\subsection{Cálculo do Valor Presente sem Flexibilidade}

Com as entradas e saídas de recursos financeiros do projeto definidas, assim como o custo de capital, chegou-se ao VP e ao VPL, ao deduzir investimentos iniciais, sem flexibilidade gerencial do projeto. Esse mesmo VP foi esquematizado na árvore binomial. O resultado encontrado na avaliação da implantação de MDL sem flexibilidade considerando um custo do capital de $27,65 \%$ ao ano foi um VPL de $€ 179.785,12$, evidenciado no fluxo de caixa que consta no anexo 1.

O VP no instante zero do projeto após a implantação do MDL com o mesmo custo de capital WACC igual a 27,65\% ao ano, também está apresentado no mesmo fluxo de caixa e seu valor foi de €424.276,11.

\subsection{Cálculo da Volatilidade Consolidada do Projeto}

Para o cálculo da volatilidade consolidada do projeto, levou-se em consideração o preço da $\mathrm{TCO}_{2} \mathrm{e}$ no mercado VER.

Revista Produção Online, Florianópolis, SC, v.15, n. 3, p. 1049-1079, jul./set. 2015. 
O risco considerado neste estudo foi a volatilidade dos preços da $\mathrm{TCO}_{2} \mathrm{e}$ no mercado VER, que é o desvio padrão dos mesmos preços históricos analisados.

O preços históricos da $\mathrm{TCO}_{2} \mathrm{e}$ no mercado VER seguiram uma distribuição estatística do tipo beta com os seguintes parâmetros: $\alpha=8,5354$ (parâmetro da distribuição), $\beta=2,3981$ (parâmetro da distribuição), $A=-€ 3,43$ (limite inferior para o intervalo de dados do referido mercado) e $B=7,32$ (limite superior para o intervalo de dados do referido mercado). A distribuição dos preços históricos da $\mathrm{TCO}_{2} \mathrm{e}$ pode ser confirmada na figura 6.1.

Os parâmetros da distribuição estatísticas para o preço da $\mathrm{TCO}_{2} \mathrm{e}$ foram utilizados como dados de entrada no modelo de simulação na obtenção da volatilidade consolidada do projeto.

Conforme Samuelson (1965) pode-se combinar as incertezas (riscos) do projeto com a Simulação de Monte Carlo, o que permiti obter estimativas da volatilidade desse mesmo projeto. Assim foi realizada a combinação dos riscos que foi traduzida na volatilidade única do projeto.

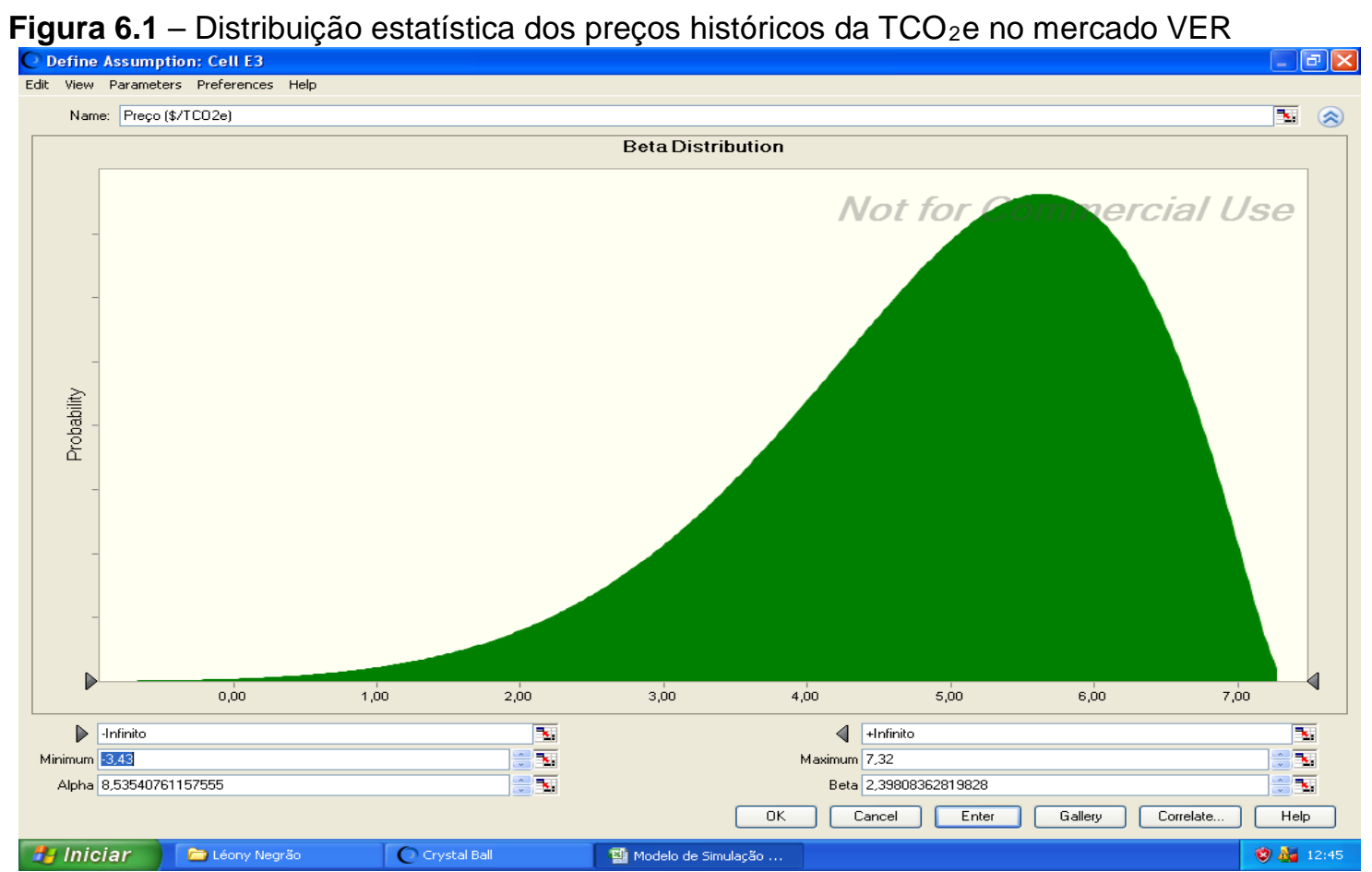

Fonte: Dos Autores (2013) 
Utilizando o programa Crystal Ball 11.1.1.0.00, e por meio da Simulação de Monte Carlo, foi calculado a volatilidade consolidada do projeto. O programa em 20.000 iterações simulou no ano zero de implantação do projeto de MDL um VP do projeto sem flexibilidade de €424.276,11 no mercado VER. Sendo que a cada nova interação os valores líquidos e futuros do fluxo de caixa do projeto mudam em função das gerações dos preços da $\mathrm{TCO}_{2} \mathrm{e}$ simulados.

O desvio padrão obtido como resultado da Simulação de Monte Carlo foi $28,70 \%$ para o valor do projeto gerado no mercado VER, e pode ser verificado no quadro 6.1 .

Quadro 6.1 - Resumo dos resultados da Simulação de Monte Carlo no mercado VER

\begin{tabular}{|r|r|}
\hline Forecast Name & VPL \\
\hline Trials & 20.000 \\
\hline Mean & $€ 146.769,78$ \\
\hline Median & $€ 149.211,31$ \\
\hline Mode & \\
\hline Standard Deviation & $€ 42.122,08$ \\
\hline Variance & $€ 1.774 .269 .521,97$ \\
\hline Skewness & $-0,3491$ \\
\hline Kurtosis & 3,06 \\
\hline Coeff. of Variability & 0,2870 \\
\hline Minimum & $€(47.351,20)$ \\
\hline Maximum & $€ 269.627,84$ \\
\hline Mean Std. Error & $€ 297,85$ \\
\hline $5 \%$ & $€ 73.162,41$ \\
\hline $95 \%$ & $€ 211.491,25$ \\
\hline
\end{tabular}

Fonte: Dos Autores (2013)

\subsection{Modelagem da Árvore Binomial}

Conhecido o valor da volatilidade consolidada do projeto, foi construída a árvore de eventos, considerando o modelo binomial, sem as flexibilidades gerenciais. Os investimentos iniciais para implantação do MDL, em Teoria de Opções Reais o preço de exercício, é representado por $X$ e seu valor foi de $€ 182.283,52$. O valor do ativo subjacente, ou seja, o valor presente do projeto, que nesta pesquisa foi representado por VP no instante zero, obtido por intermédio do FCD tradicional, é igual a €424.276,11, no mercado VER. 
De posse desses valores e com o auxílio de planilhas eletrônicas, conforme apresentado por Copeland e Antikarov (2001), foi construída a árvore binomial, sem a OR considerada neste trabalho, o que pode ser averiguada no quadro 6.2. Essa árvore foi a base sobre a qual se inseriu a OR de Diferimento ou Adiamento.

Quadro 6.2 - Árvore binomial para o VP do projeto de $€ 424.276,11$

\begin{tabular}{|c|c|c|c|c|c|c|}
\hline \multicolumn{4}{|c|}{ ÁRVORE DE EVENTOS PARA O ATIVO SUBJACENTE } & & & \\
\hline Período & $\overline{0}$ & $\overline{1}$ & 2 & 3 & 4 & 5 \\
\hline 0 & $€ 424.276,11$ & $€ 565.315,76$ & $€ 753.240,41$ & $€ 1.003 .635,76$ & $€ 1.337 .268,59$ & $€ 1.781 .809,05$ \\
\hline 1 & & $€ 318.424,20$ & $€ 424.276,11$ & $\begin{array}{ll}€ 565.315,76 \\
\end{array}$ & $\begin{array}{ll}€ & 753.240,41\end{array}$ & $€ 1.003 .635,76$ \\
\hline 2 & & $€$ & $€ 238.981,09$ & $\begin{array}{ll}E & 318.424,20\end{array}$ & $\begin{array}{ll}€ & 424.276,11\end{array}$ & $€ \quad 565.315,76$ \\
\hline 3 & & $€$ & $€$ & $\begin{array}{ll}€ € & 179.358,11\end{array}$ & € 238.981,09 & $\begin{array}{ll} & 318.424,20\end{array}$ \\
\hline 4 & & $€$ & $€$ & $€$ & $€ 134.610,37$ & $€ \quad 179.358,11$ \\
\hline 5 & & $€$ & $€$ & $€$ & $€$ & $\begin{array}{ll} & 101.026,66\end{array}$ \\
\hline
\end{tabular}

Fonte: Dos Autores (2013)

\subsection{Cálculo da Opção de Diferimento no Projeto e Modelagem da Árvore com a Opção}

A Opção de Adiamento foi caracterizada pelo exercício da flexibilidade gerencial de postergar (ou diferir) o início de implantação do projeto. Esse evento foi realizado durante o período de 2009 a 2013. O cenário dos preços da $\mathrm{TCO}_{2} \mathrm{e}$ comercializada no mercado VER que viabiliza a implantação de mecanismo permitiu o exercício ou não da OR.

O exercício da opção de realizar os investimentos na implantação do projeto ou de diferir os investimentos dar-se-á quando o cenário do preço da $\mathrm{TCO}_{2} \mathrm{e}$ for tal que justifique pagar por um prêmio de Opção no momento adequado para investir os $€ 182.283,52$.

O quadro 6.3 apresenta o valor da opção em cada instante do horizonte de tempo analisado. Os valores da OR de Diferimento foi calculado segundo a equação 29 na direção do período 5 (cinco) ao período 0 (zero), baseada nos valores obtidos na árvore binomial para o ativo subjacente. 


$$
C_{0}=\frac{\left[p C_{u}+(1-p) C_{d}\right]}{\left(1+r_{f}\right)}
$$

Quadro 6.3 - Árvore binomial com valor de premio da OR de €227.663,02

\begin{tabular}{|c|c|c|c|c|c|c|}
\hline \multicolumn{4}{|c|}{ VALOR DA OPCÃO DE DIFERIMENTO } & \\
\hline Período & 0 & 1 & 2 & 3 & 4 & 5 \\
\hline 0 & $€ 396.505,82$ & $€ 524.220,03$ & $€ 690.845,35$ & $\begin{array}{ll}€ & 906.248,53 \\
\end{array}$ & $€ 1.180 .920,04$ & $€ 1.599 .525,53$ \\
\hline 1 & & $€ 303.343,82$ & $€ 404.182,70$ & $\begin{array}{ll}€ & 538.542,91 \\
\end{array}$ & $€ 717.567,72$ & $€ 1.003 .634,76$ \\
\hline 2 & & $€$ & $€ 227.663,02$ & $\begin{array}{ll}€ & 303.343,72 \\
\end{array}$ & $€ \quad 404.182,61$ & $\begin{array}{ll}€ & 565.315,48 \\
\end{array}$ \\
\hline 3 & & $€$ & $€$ & $\begin{array}{ll}€ & 170.863,78 \\
\end{array}$ & $€ 227.662,89$ & $€ \quad 318.423,20$ \\
\hline 4 & & $€$ & $€$ & $€$ & $€ \quad 128.235,47$ & $\begin{array}{ll} & 179.358,11\end{array}$ \\
\hline 5 & & $€$ & $€$ & $€$ & $€$ & $€ \quad 101.026,66$ \\
\hline
\end{tabular}

Fonte: Dos Autores (2013)

Conforme pode ser verificado no quadro 6.3, o exercício da OR, considerando os investimentos para a implantação do MDL, deverá ser exercido no instante 2, no ano de 2010, onde apresenta um valor de prêmio para a OR de €227.663,02.

\subsection{Avaliação dos investimentos}

Como percebido na realização da pesquisa, a OR de Diferimento ou Adiamento adicionou valor ao projeto de implantação do MDL na empresa objeto de estudo medido pelo VPL sem flexibilidade.

Considerando o final de cada período na avaliação de uma Opção de compra americana (nesta pesquisa OR de Adiamento ou Diferimento) sobre um ativo subjacente sujeito a risco, pressupondo-se um passo ao ano, o valor estimado da referida Opção obtida foi de €396.505,82 no instante zero, que somada ao preço de exercício, supera o seu VP no mesmo instante, o que leva a decisão de adiar a realização do investimento, como isso não exercendo a Opção. O que pode ser confirmado no quadro 6.3.

Entretanto, o projeto é considerado viável para implantação, pois apresenta um VPL positivo, o que se considera estratégico para a empresa optar pela implantação do projeto visto que no ano de 2010 houve uma agregação de valor para o mesmo. 
O quadro 6.3 evidencia o valor agregado do projeto em €227.663,02 (valor da OR) que, somado ao preço de exercício de $€ 182.283,52$, é menor que o VP do projeto no instante zero, de €424.276,11.

Como o VPL do projeto sem flexibilidade no instante zero foi positivo, $€ 179.785,12$ (VPL estático), indicando a viabilidade do mesmo à decisão de investir na implantação do MDL, exercendo a OR, no ano de 2010, possibilita agregar valor àquele VPL em €227.663,02, valor da OR, gerando um VPL expandido - VPLE - de $€ 407.448,14$, conforme a equação 22.

O quadro 6.4 apresenta um resumo das decisões de exercer ou não a Opção para o horizonte de tempo considerado na pesquisa, e que foi determinada conforme expressões 23 e 24.

Quadro 6.4 - Árvore com os tipos de decisões, enfatizando o exercício da Opção no período 2

\begin{tabular}{|c|c|c|c|c|c|c|}
\hline \multicolumn{4}{|c|}{ RESUMO DAS DECISÕES EM CADA INSTANTE } & \\
\hline Período & 0 & 1 & 2 & 3 & 4 & 5 \\
\hline 0 & adiar & adiar & adiar & adiar & adiar & adiar \\
\hline 1 & & adiar & adiar & adiar & adiar & adiar \\
\hline 2 & & & investir & adiar & adiar & adiar \\
\hline 3 & & & & investir & investir & adiar \\
\hline 4 & & & & & investir & investir \\
\hline 5 & & & & & & investir \\
\hline
\end{tabular}

Fonte: Dos Autores (2013)

\section{CONCLUSÃO}

A presente pesquisa teve como objetivo realizar a avaliação econômica da implantação do MDL em uma empresa de cerâmica vermelha, à luz de metodologia da TOR, em que foi considerada a Opção de Diferimento ou Adiamento.

A avaliação considerou um período estimado de validação do Protocolo de Quioto de 2008 a 2012. Entretanto, a consultoria realizada para implantação do mecanismo estimou reduções de $\mathrm{CO}_{2}$ durante o período de 2008 a 2017. Os investimentos e despesas de consultoria, assim como as receitas provenientes da venda dos créditos de carbono foram projetados conforme aquele intervalo de tempo por um prazo de 5 anos.

Considerou-se a estrutura de capital constituída somente por recursos Revista Produção Online, Florianópolis, SC, v.15, n. 3, p. 1049-1079, jul./set. 2015. 
próprios, como de fato ocorreu, e foi obtido o valor presente do projeto sem flexibilidade operacional.

Com base nessas considerações foi realizada a inclusão da OR que representou a quantificação da flexibilidade gerencial traduzida e associada a Opção de Adiamento ou não dos investimentos necessários à implantação do MDL para geração e solicitação dos créditos de carbono no mercado VER.

Utilizou-se como incertezas (riscos) para o exercício ótimo da Opção, os preços da tonelada de $\mathrm{CO}_{2} \mathrm{e}$ no referido mercado.

A análise de Opções Reais por meio da Opção de Diferimento ou Adiamento em uma empresa de cerâmica vermelha possibilitou a disponibilização de informações coerentes contribuindo para as decisões gerenciais de viabilidade econômica, algo ainda inédito no setor, levando a exprimir o pleno atendimento do objetivo da pesquisa.

Os dados estimados permitiram avaliar a implantação do projeto de MDL na empresa cerâmica de acordo com o método proposto. Foi obtido o VP sem flexibilidade, e por meio da Simulação de Monte Carlo consolidou-se a volatilidade do projeto. Este dois dados somados à taxa livre de risco, com análise discreta, possibilitaram desenvolver uma árvore de eventos sem flexibilidade. A essa árvore foi incorporada a flexibilidade correspondente a Opção de Diferimento ou Adiamento.

Os resultados traduzidos em aumento de valor da implantação do MDL comparado ao valor da empresa sem o projeto com a inserção da Opção Real de Diferimento, demonstram a importância da utilização da metodologia da TOR na avaliação das flexibilidades dos projetos, a qual caracterizou a atenção a ser transmitida às flexibilidades gerenciais, que no caso deste estudo, somadas à avaliação tradicional por FCD, possibilitou a obtenção do valor adequado para o projeto.

O valor expandido do projeto, ou o VPL incluindo a flexibilidade gerencial de realizar o investimento na implantação do MDL ou de Deferir o investimento foi de $€ 330.555,96$ considerando o valor da OR obtida de €170.863,76 em relação ao VPL sem flexibilidade gerencial de €159.692,20, o que agregou valor ao projeto na justificativa de viabilidade da realização do investimento ou preço de exercício de $€ 182.283,20$ para implantação do projeto de MDL.

Revista Produção Online, Florianópolis, SC, v.15, n. 3, p. 1049-1079, jul./set. 2015. 
Importante ressaltar a contribuição da pesquisa no sentido de suprir os empresários do setor, disponibilizando informações confiáveis e compatíveis com suas realidades, contribuindo com o processo de tomada de decisão empresarial possibilitando a perseverança sustentável dos empreendimentos.

Outra contribuição está no fato de que a avaliação desse tipo de empreendimento é peculiar, sendo que no setor de cerâmica industrial, a implantação de MDL objetivando a comercialização de créditos de carbono, sendo avaliado por Teoria de Opções Reais, ainda não existia. O que torna um marco para o setor e que poderá estender-se para outras empresas do mesmo segmento dentro ou fora do Estado do Pará.

O mesmo ocorre com a utilização da Opção de Diferimento, onde a aplicação em mais de um período para valoração do prêmio de Opção por meio do Modelo Binomial, conforme realizado nesta pesquisa se configurou como algo desafiador diante da indisponibilidade de referências bibliográficas tal falto reforça a contribuição, neste campo, desta pesquisa.

E no âmbito acadêmico a utilização deste estudo pode servir como fonte de consulta na possibilidade de abertura de diversos trabalhos futuros relacionados ao tema e ao setor de aplicação.

Enfim, o método proposto contribuiu para o desenvolvimento e utilização da teoria em um ambiente de risco onde convencionou coerentemente os parâmetros necessários para a geração e obtenção dos resultados. Reforçando a aplicação do método considerando as particularidades de cada caso, mas que podem seguir as etapas ratificadas pelo caso demonstrado na pesquisa.

Como trabalhos futuros, na avaliação de implantação do MDL em empresas cerâmicas, propõem-se o estudo de outras OR simples e ou compostas equivalentes à flexibilidade gerencial para que se possam comparar os diversos resultados obtidos aos determinados neste artigo.

O estudo para a utilização de uma volatilidade variável anual ao longo de toda a vida do projeto, também pode ser estudada por outros trabalhos. Assim como, a continuidade deste estudo ao fim do período analisado no mesmo, considerando outras estimativas de reduções de emissão de $\mathrm{CO}_{2}$ que deverão ser projetadas conforme condições estruturais da referida empresa, ainda por meio da implantação 
do MDL.

Finalmente, a inclusão na avaliação da implantação do MDL o mercado de Certificado de Redução de Emissões - CER - considerando os investimentos necessários às fases para solicitação dos créditos de carbono e o preço da $\mathrm{TCO}_{2} \mathrm{e}$ nesse mercado.

\section{REFERÊNCIAS}

ASSAF NETO, A. Contribuição ao estudo de avaliação de empresas no Brasil: uma aplicação prática. Ribeirão Preto. Tese (Livre Docência). Faculdade de Economia, Administração e Contabilidade. Universidade de São Paulo. São Paulo, 2004.

BRANDÃO, L. E. T. Uma aplicação da teoria das opções reais em tempo discreto para avaliação de uma concessão rodoviária no Brasil. Tese de Doutorado - DEI Pontifícia Universidade Católica. Rio de Janeiro, 2002.

BRANDÃO, L. E. T.; DYER, J. S.; HAHN, W. J. Using binomial decision trees to solve real option valuation problems. Decision Analysis, v.0, p. 1-20, 2005a. DOI:

http://dx.doi.org/10.1287/deca.1050.0040

COPELAND, T.; ANTIKAROV, V. Opções reais: um novo paradigma para reinventar a avaliação de investimentos. Rio de Janeiro: Campus, 2001.

COX, J.; ROSS, S.; RUBINSTEIN, M. Option pricing: a simplified approach. Journal of Financial Economics, v. 7, n. 3, p. 229-248, 1979.DOI: http://dx.doi.org/10.1016/0304405X(79)90015-1

CLEAN DEVELOPMENT MECHANISM. Project design document form. São Paulo, 2008.

DIAS, M.A.G.. Valuation of exploration and production assets: an overview of real option models. Journal of Petroleum Science and Engineering, v. 44, n. 1-2, p. 93-114, 2004. DOI: http://dx.doi.org/10.1016/j.petrol.2004.02.008

DIXIT, A.K.; PINDYCK, R. S. Expandability, reversibility, and optimal capacity choice. nber working paper series. Working Paper 6373. 28 p. National Bureau of Economics Research, Cambridge, MA, USA, January 1998.

HULL, John C. Options, futures and other derivatives. 6. ed. New Jersey: Prentice Hall, 2005.

LOPES, I. V. O mecanismo de desenvolvimento limpo - MDL: guia de orientação - Rio de Janeiro: Fundação Getulio Vargas, 2002. 90p. Disponível em: <http://www.mct.gov.br >. Acesso em 30 de março de 2008.

MARTELANC, R. Passos para avaliação de uma empresa de pequeno porte pelo método do valor presente do fluxo de caixa constante. Temática Contábil, boletim 46/98. p. 1-6, 1998.

MERLIN, S. Mudanças Climáticas: desafios e oportunidades. Ecológica Assessoria. 2007. Revista Produção Online, Florianópolis, SC, v.15, n. 3, p. 1049-1079, jul./set. 2015. 
MONTEIRO, R. C. Contribuições da abordagem de avaliação de opções reais em ambientes econômicos de grande volatilidade - uma ênfase no cenário latino-americano. 2003. 200 f. Dissertação (Mestrado em Controladoria e Contabilidade) - Faculdade de Economia, Administração e Contabilidade, Universidade de São Paulo, São Paulo.

PORTAL BRASIL. Caderneta de poupança: índices diários. Disponível em:

<HTTP://www.portalbrasil.net>. Acessado em 04 de dezembro de 2014 .

RIBEIRO, M. S. O tratamento contábil dos créditos de carbono. Tese de livre docência. Faculdade de Economia, Administração e Contabilidade, Universidade de São Paulo. São Paulo, 2005.

ROSS. S. A.; WESTERFIELD, R. W.; JAFFE, J. F. Administração financeira corporate finance. São Paulo, Brasil: Editora Atlas, 1995.

SHARPE, W. Portfolio theory and capital markets. New York: McGraw Hill, 1970.

SILVA NETO, L. A. Opções: do tradicional ao exótico. 2. ed. São Paulo: Atlas, 1996.

TRIGEORGIS, L. Real option: managerial flexibility and strategy in resource allocation. Cambridge, MA: The MIT Press, 1996.

WOILER, S., MATHIAS, W. F. Projetos: planejamento, elaboração e análise. São Paulo: Atlas, 1997.

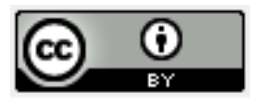

Artigo recebido em 07/12/2014 e aceito para publicação em 11/12/2014 DOI: http://dx.doi.org/ 10.14488/1676-1901.v15i3.1948 
Apêndice 1 - Fluxo de caixa da cerâmica Gama LTDA no mercado VER

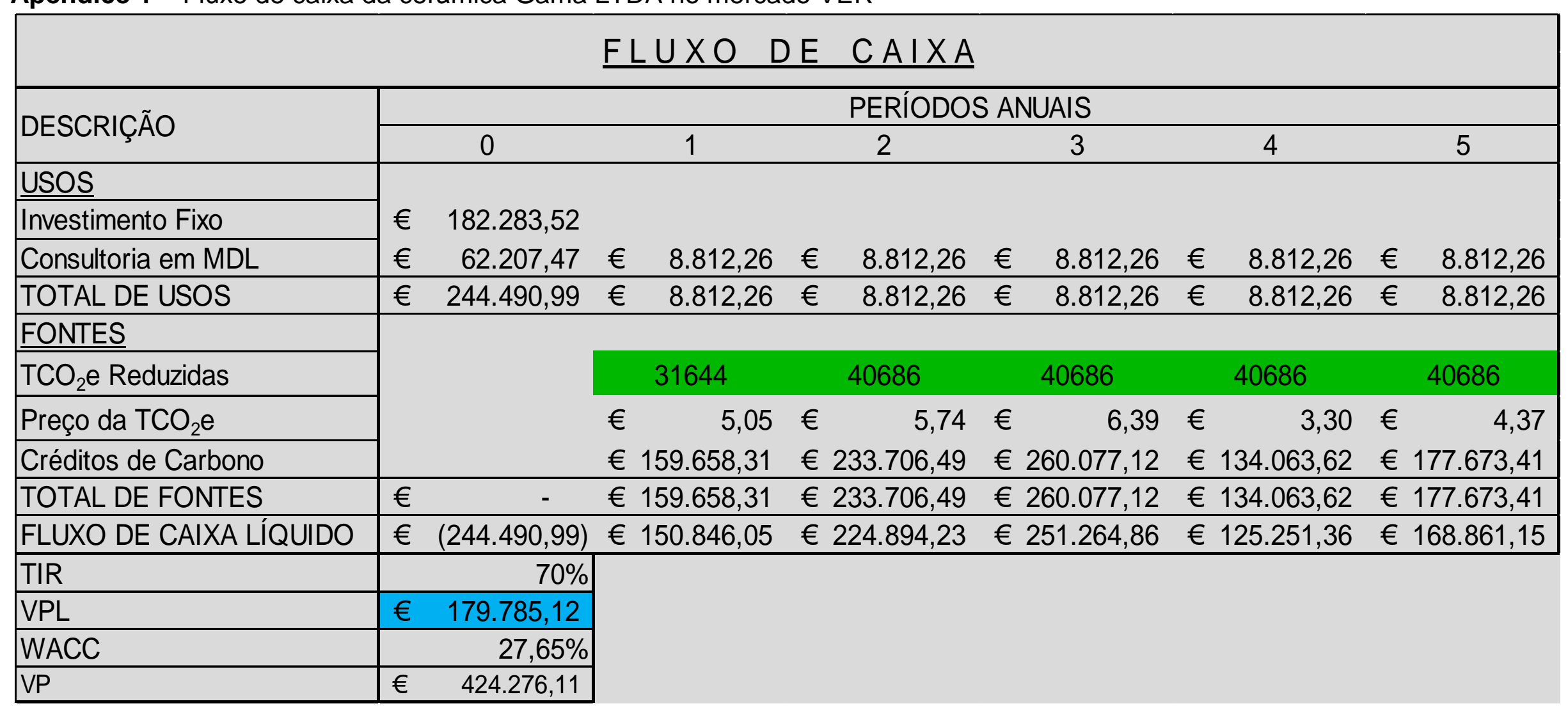

Fonte: Dos Autores (2013) 